\title{
Dibenzazepine Agents in Epilepsy: How Does Eslicarbazepine Acetate Differ?
}

\author{
Charlotte Lawthom (D) J Jukka Peltola - Rob McMurray • \\ Emma Dodd · Vicente Villanueva
}

Received: August 6, 2018 / Published online: September 24, 2018

(C) The Author(s) 2018

\begin{abstract}
Carbamazepine (CBZ), oxcarbazepine (OXC), and eslicarbazepine acetate (ESL) belong to the dibenzazepine family of antiepileptic drugs and are all thought to primarily act as sodium channel blockers (SCBs). However, ESL is structurally distinct from $\mathrm{CBZ}$ and $\mathrm{OXC}$, resulting in differences in metabolism, pharmacokinetics, and pharmacodynamics. Despite a lack of direct comparative data, evidence for potential differences in effectiveness and tolerability within the dibenzazepine family has emerged from studies in which patients being treated with one dibenzazepine agent have received adjunctive
\end{abstract}

Enhanced digital features To view enhanced digital features for this article go to https://doi.org/10.6084/ m9.figshare.7082696.

C. Lawthom $(\bowtie)$

Aneurin Bevan University Health Board,

Newport, UK

e-mail: charlotte.lawthom2@wales.nhs.uk

J. Peltola

Tampere University Hospital, Tampere, Finland

R. McMurray · E. Dodd

Eisai Europe Ltd, Hatfield, Hertfordshire, UK

V. Villanueva

Refractory Epilepsy Unit, Neurology Service,

Hospital Universitario y Politécnico La Fe, Valencia, Spain treatment with another (having achieved insufficient seizure control with the first) or have transitioned from one dibenzazepine agent to another because of lack of effectiveness or poor tolerability. Most of these studies have been conducted in the real-world clinical practice setting. ESL has been shown to be effective as adjunctive therapy in patients who have previously achieved inadequate seizure control with CBZ, indicating that the use of different dibenzazepine agents in combination can provide additive effectiveness benefits, which may reflect underlying differences in their mechanisms of action. Similarly, ESL monotherapy can be effective in patients who have switched from another dibenzazepine, such as CBZ or $\mathrm{OXC}$, because of inadequate efficacy. There is also considerable evidence to demonstrate that patients transitioning from OXC or CBZ to ESL as a result of adverse events experience improvements in tolerability, which may also be associated with improvements in quality of life, alertness, and/or lipid profiles. Current evidence therefore demonstrates that ESL differs from other dibenzazepine agents in terms of effectiveness and tolerability.

Funding: Eisai Ltd.

Keywords: Antiepileptic drug; Carbamazepine; Dibenzazepine; Epilepsy; Eslicarbazepine acetate; Oxcarbazepine 


\section{INTRODUCTION}

Sodium channel blockers (SCBs) have played a central role in the pharmacological management of focal and generalized tonic-clonic seizures since phenytoin was first used as an antiepileptic drug (AED) in 1936 [1]. In addition to phenytoin, AEDs that are primarily SCBs in their mechanism of action include lamotrigine (LTG), lacosamide, rufinamide, and the dibenzazepine carboxamide family of AEDs, comprising carbamazepine (CBZ), oxcarbazepine (OXC), and eslicarbazepine acetate (ESL) [1]. Despite sharing a common mechanistic targetthe voltage-gated sodium channel-SCBs differ in their specific mechanisms of action. For example, phenytoin, CBZ, and $\mathrm{OXC}$ are thought to block the fast inactivation of sodium channels, whereas lacosamide and ESL are thought to act on the slow inactivated state of sodium channels [1-3]. Such mechanistic variations may underlie the observed differences in efficacy of SCBs, and help to explain why SCBs may show additive effects when used in combination, compared with when used as single agents alone [1]. Furthermore, SCBs differ markedly from each other in their tolerability/ safety profiles, particularly in terms of their variable propensity for enzyme induction $[1,4]$.

The objective of this article is to review current evidence for differences in effectiveness and tolerability between ESL and other agents within the dibenzazepine family of AEDs, and to discuss the implications of these differences in clinical practice. This article is based on previously conducted studies. All procedures performed in studies conducted by the authors involving human participants were in accordance with the ethical standards of the institutional and/or national research committee and with the 1964 Helsinki declaration and its later amendments or comparable ethical standards; informed consent was obtained from all individual participants included in these studies. The article also contains studies with human participants or animals that were not performed by any of the authors.

\section{OVERVIEW OF THE DIBENZAZEPINE FAMILY}

CBZ was discovered by chance in the 1950 s $[5,6]$ and, over subsequent decades, became widely accepted as the first drug of choice for focal epilepsy [7] and the standard comparator for European regulatory studies in newly diagnosed epilepsy [1]. OXC, a keto analogue of CBZ [8], was introduced in the 1980s, with the aim of improving the pharmacokinetic profile and tolerability of CBZ [6]. In the 1990s, esters of its major metabolite were synthesized in order to provide further improvements in pharmacokinetics and tolerability, which resulted in the development of ESL $[6,9]$.

\section{Pharmacology and Potential Clinical Correlates}

CBZ, OXC, and ESL all have a dibenzazepine nucleus bearing the 5-carboxamide substitute, but ESL is structurally distinct from CBZ and OXC at the 10,11-position (Fig. 1) [10, 11], resulting in differences in metabolism, pharmacokinetics, and pharmacodynamics (Table 1) [15]. Unlike CBZ, ESL is not metabolized to carbamazepine-10,11-epoxide, the metabolite which may play a major role in causing adverse effects, such as drowsiness, dizziness, and diplopia $[6,9,16,17]$. CBZ's tolerability is further affected by its potent enzyme induction properties, which reduce the duration and action of many drugs [4] and may additionally contribute to the development of comorbidities, including vascular disease, osteoporosis, and sexual dysfunction, via effects on enzymes involved in endogenous metabolic pathways [4, 18].

ESL's metabolism also differs from that of OXC. OXC rapidly undergoes hydroxylation to an active 10-monohydroxy derivative, comprising an enantiomeric mixture of $(S)$-licarbazepine ("eslicarbazepine") and (R)licarbazepine (in a ratio of $4: 1$ ), with a small amount of parent compound remaining in the plasma $[8,11,19]$. ESL is stereoselectively metabolized primarily to (S)-licarbazepine, which accounts for approximately $94 \%$ of 

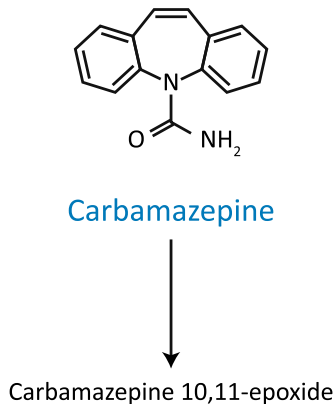

Carbamazepine 10,11-epoxide
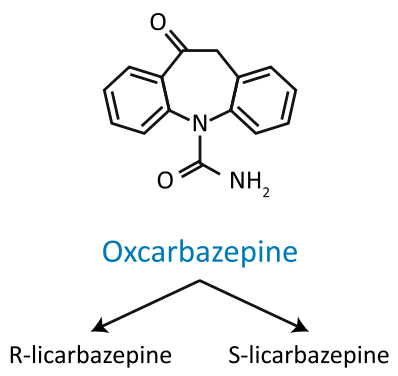

R-licarbazepine:S-licarbazepine
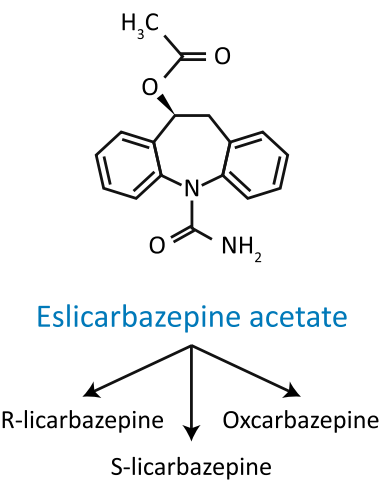

R-licarbazepine:S-licarbazepine $1: 19$
Fig. 1 Chemical structure and main metabolic pathways of CBZ, OXC, and ESL. CBZ carbamazepine, ESL eslicarbazepine acetate, OXC oxcarbazepine. Reprinted by permission from Springer Nature, Neurotherapeutics (Eslicarbazepine acetate (BIA 2-093)), Luis Almeida, Patrício Soares-da-Silva, 2007

Table 1 Summary comparison of metabolism, pharmacokinetics, and pharmacodynamics of CBZ, OXC, and ESL $[3,11-14]$

CBZ OXC ESL

Metabolism

$\begin{array}{llll}\begin{array}{l}\text { Route } \\ \begin{array}{l}\text { Primary } \\ \text { biotransformation } \\ \text { pathway }\end{array}\end{array} & \begin{array}{c}\text { Epoxide biotransformation to } \\ \text { CBZ 10,11-epoxide }\end{array} & \begin{array}{c}\text { Hepatic } \\ \text { Cytosolic reduction to } \\ 10 \text {-monohydroxy derivative }\end{array} & \begin{array}{c}\text { Hydrolysis } \\ \text { Pharmacokinetics }\end{array} \\ \begin{array}{l}\text { Time to maximum } \\ \text { plasma concentration }\end{array} & 12 \mathrm{~h} & 4.5 \mathrm{~h} & 2-3 \mathrm{~h} \\ \begin{array}{l}\text { Time to steady state } \\ \text { Half-life of active }\end{array} & 1-2 \text { weeks } & & 4-5 \text { days } \\ \text { metabolite } & 6 \mathrm{~h} & 2-3 \text { days } & 20-24 \mathrm{~h}\end{array}$

Pharmacodynamics

\begin{tabular}{llll} 
Main active metabolite & CBZ 10,11-epoxide & 10-Monohydroxy derivative ${ }^{\mathrm{a}}$ & $(S)$-Licarbazepine \\
$\begin{array}{l}\text { Primary mechanism of } \\
\text { action }\end{array}$ & $\begin{array}{c}\text { Fast inactivation of voltage- } \\
\text { gated sodium channels }\end{array}$ & $\begin{array}{c}\text { Fast inactivation of voltage- } \\
\text { gated sodium channels }\end{array}$ & $\begin{array}{c}\text { Slow inactivation of voltage- } \\
\text { gated sodium channels }\end{array}$ \\
\hline
\end{tabular}

$C B Z$ carbamazepine, ESL eslicarbazepine acetate, $O X C$ oxcarbazepine

${ }^{a}$ An enantiomeric mixture of $(S)$-licarbazepine and $(R)$-licarbazepine in a ratio of $4: 1$

plasma drug exposure following oral administration of ESL, with $(R)$-licarbazepine and oxcarbazepine accounting for approximately $5 \%$ and less than $1 \%$, respectively (ratio of
$(S)$-licarbazepine to $(R)$-licarbazepine, approximately 19:1; Fig. 1) [11, 19]. Importantly, this stereoselective metabolism of ESL avoids the early peaks in (R)-licarbazepine and OXC 
concentrations observed in plasma and cerebrospinal fluid following immediate-release OXC administration, which are thought to be associated with OXC-related side effects, such as dizziness and headache [19]. Consequently, ESL may be associated with fewer neurological/psychiatric side effects than OXC $[13,14,20]$.

Evidence from therapeutic drug monitoring databases has demonstrated that ESL is associated with fewer interactions with other AEDs than CBZ and OXC [21]. The pharmacokinetic profile of ESL was shown to be unaffected by enzyme-inducing AEDs and valproate, and ESL did not affect the metabolism of LTG; by contrast, CBZ clearance was found to be increased by enzyme-inducing AEDs and valproate, and LTG clearance was increased by both OXC and CBZ [21]. Furthermore, once-daily dosing of ESL may improve the likelihood of adherence to ESL treatment in comparison with $\mathrm{CBZ}$ and $\mathrm{OXC}$, since adherence to a once-daily AED dosing regimen has been shown to be significantly better than to a twice- or three-times daily dosing regimen [22].

\section{Pharmacodynamic Differences}

ESL differs pharmacodynamically from CBZ, since ESL is thought to act primarily by enhancing the slow inactivation of voltagegated sodium channels, whereas CBZ is thought to alter the fast inactivation of these channels [3]. As previously mentioned, although OXC and ESL share a common active metabolite (eslicarbazepine), this accounts almost exclusively for ESL's pharmacodynamic effect, whereas it is the monohydroxy derivative of OXC (comprising a substantial proportion of $(R)$-licarbazepine as well as eslicarbazepine) that accounts for its pharmacodynamic effect (Fig. 1) [8, 11, 19]. The apparent affinity of eslicarbazepine for voltagegated sodium channels in the inactivated and resting states is considerably lower than that of CBZ and $(R)$-licarbazepine, suggesting that eslicarbazepine has enhanced selectivity for inhibiting rapidly firing active "epileptic" neurons [23-26]. This may in part explain the observed efficacy of ESL in the presence of CBZ resistance in experimental models of epilepsy, which have demonstrated significant add-on effects when ESL is used in combination with CBZ $[27,28]$. These pharmacodynamic differences are thought to influence the clinical effectiveness and tolerability of the members of the dibenzazepine family, CBZ, OXC, and ESL.

\section{DIFFERENCES IN EFFECTIVENESS: EVIDENCE FROM CLINICAL STUDIES}

Few clinical studies have directly compared the efficacy and tolerability of members of the dibenzazepine family. Results from the Standard And New Antiepileptic Drugs (SANAD) study suggested a non-significant advantage for CBZ compared with OXC [7]. ESL was not included as it was not approved at the time SANAD was conducted. In a phase III randomized controlled trial, ESL was shown to be noninferior to controlled-release CBZ (CBZ-CR) as monotherapy for patients with newly diagnosed focal epilepsy [29]. Seizure freedom rates during the entire 6-month evaluation period for the per protocol population (the primary endpoint of the trial) were $71.1 \%$ with ESL and $75.6 \%$ with CBZ-CR [29].

Despite the lack of direct comparative data, evidence for potential differences in effectiveness (and tolerability) within the dibenzazepine family has emerged from studies in which patients have received adjunctive treatment with a dibenzazepine AED. These patients had achieved insufficient seizure control with another AED, or were transitioned from one dibenzazepine agent to another, because of lack of effectiveness or poor tolerability. The majority of this evidence-as outlined below-has emerged from the real-world setting, highlighting the importance of clinical practice studies in further complementing evidence obtained from clinical trials.

A post hoc analysis was conducted of 1049 patients enrolled in three phase III, multicenter, double-blind, randomized, placebo-controlled trials, in which adults with focal seizures, despite treatment with one to three AEDs, received adjunctive treatment with ESL [30]. Efficacy was compared between patients who 


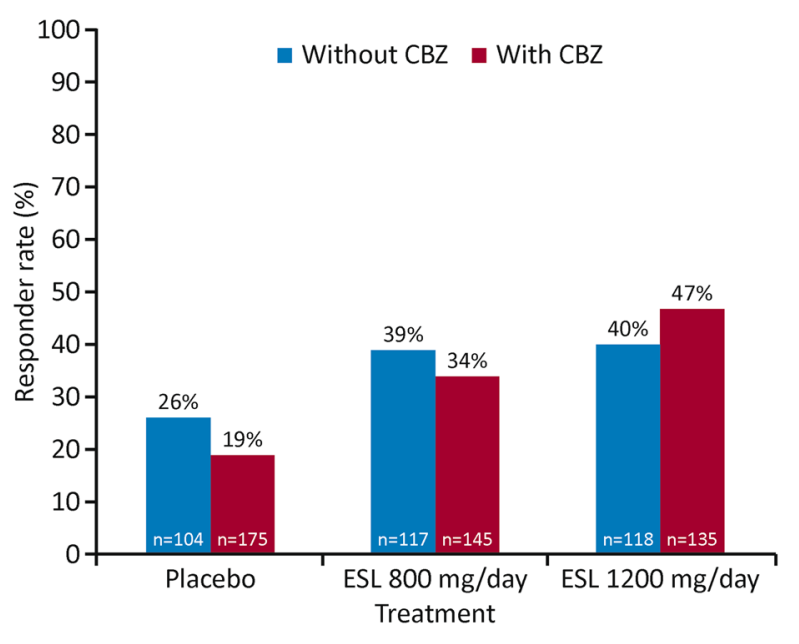

Fig. 2 Responder rates in patients included in the ESL phase III adjunctive therapy trials who were not receiving $\mathrm{CBZ}$ or who were receiving CBZ. Response was defined as at least $50 \%$ seizure frequency reduction from baseline [30]. CBZ carbamazepine, ESL eslicarbazepine acetate

did $(n=611)$ and did not $(n=438)$ receive CBZ as a concomitant AED [30]. Seizure frequency over a 12-week maintenance period (the primary endpoint) was significantly reduced, compared with placebo, in patients treated with ESL 800 and $1200 \mathrm{mg} /$ day, not only when administered without CBZ $(p<0.01$ and $p<0.05$, respectively) but, interestingly, also when administered with $\mathrm{CBZ}(p<0.01$ and $p<0.0001$, respectively) [30]. In patients not receiving CBZ, responder rates (response defined as at least $50 \%$ seizure frequency reduction) were $26 \%$ with placebo, $39 \%$ with ESL $800 \mathrm{mg} /$ day, and $40 \%$ with $1200 \mathrm{mg} /$ day (Fig. 2) [30]. In those receiving CBZ, responder rates were $19 \%$ with placebo, $34 \%$ with ESL $800 \mathrm{mg} /$ day and $47 \%$ with ESL $1200 \mathrm{mg} /$ day (Fig. 2) [30]. These data suggest that ESL was efficacious in both subsets of patients, with or without concomitant CBZ. Patients who did not achieve seizure control with CBZ may still benefit from either switching to or adding in adjunctive ESL.

These findings are supported by the results of real-world clinical practice studies. The ESLIBASE study was a 1-year, multicenter, retrospective, non-interventional study, which evaluated the long-term efficacy and safety of adjunctive ESL therapy in 327 patients with focal epilepsy in a clinical practice setting in Spain [31]. Sixty patients included in the ESLIBASE study were being treated with CBZ at the start of the study [31]. Of these, 45 were transitioned from CBZ to ESL (because of lack of efficacy $[n=28]$ and tolerability problems [ $n=17$ ] with CBZ; CBZ to ESL ratio, $1: 1.5)$ and 15 were treated with both AEDs [31]. Among those who transitioned from CBZ to ESL $(n=45), 13.3 \%$ were seizure free during the 3 months prior to inclusion [31]. After 12 months of ESL treatment, $11.4 \%$ of patients were seizure free, $38.7 \%$ had responded to ESL treatment (response defined as at least 50\% seizure frequency reduction from baseline), and $20.0 \%$ had experienced worsening of seizures [31]. Similarly, 50 patients were receiving OXC before the start of the study, of whom 48 were transitioned to ESL (because of adverse events [AEs; $n=26$ ] and lack of efficacy [ $n=22$ ]; OXC to ESL ratio, 1:1) and two were treated with both AEDs. Among patients who transitioned from OXC to ESL $(n=48), 16.7 \%$ were seizure free in the 3 months prior to inclusion [31]. After 12 months of ESL treatment, $31.3 \%$ were seizure free, $45.9 \%$ were responders, and $16.7 \%$ had experienced worsening of seizures [31].

A single-center, observational, descriptive, cross-sectional study investigated the effectiveness and safety/tolerability of ESL in 61 consecutive patients with drug-resistant epilepsy over a mean follow-up duration of 4.7 months [32]. These included 13 patients who transitioned overnight from CBZ to ESL (CBZ to ESL ratio, $1: 1$ to $1: 1.3$; up to a maximum ESL dose of $1600 \mathrm{mg} /$ day) and 12 patients who transitioned overnight from OXC to ESL (OXC to ESL ratio, 1:1.1) [32]. Eight of the 13 patients who transitioned from CBZ were followed up for more than 3 months after initiating ESL and the median number of seizures/month increased by $33.3 \%$ in these patients ( $p=$ not significant) [32]. In contrast, 11 of the 12 patients who transitioned from OXC were followed up for more than 3 months and the median number of seizures/month decreased by $66.7 \%$ in these patients $(p=0.017)$ [32].

The EARLY-ESLI study was a multicenter, retrospective, 1-year, observational study conducted in 16 hospitals in Spain, which included 
253 patients with focal epilepsy, aged 18 years or over, in whom ESL treatment was initiated because of failure with the first AED monotherapy [33]. The primary reasons for ESL initiation were lack of efficacy $(n=157)$ and tolerability problems $(n=74)$ with the first AED monotherapy [33]. A total of 28 patients converted from CBZ monotherapy to ESL monotherapy at some point during follow-up. Three of four patients who converted to ESL because of lack of efficacy with CBZ were seizure free at 1 year [33]. Similarly, 12 patients converted from OXC monotherapy to ESL monotherapy, and one of four patients who did so because of lack of efficacy was seizure free at 1 year [33].

\section{Differences in Effectiveness: Summary}

Despite limited direct head-to-head data, ESL has been shown to be effective as adjunctive therapy in patients who have previously achieved inadequate seizure control with CBZ [30]. This indicates that the use of different dibenzazepine agents in combination can provide additive effectiveness benefits, which may reflect underlying differences in the mechanisms of action of this family of AEDs $[1,27,28]$. Similarly, this may in part explain why ESL monotherapy can be effective in patients who have switched from another dibenzazepine, such as CBZ or OXC, because of inadequate efficacy [31-33]. Overall, the evidence outlined here demonstrates that patients who have not achieved adequate seizure control with CBZ or OXC may experience improvement in seizure control with ESL, when used either as an adjunctive treatment or as monotherapy.

\section{TOLERABILITY PROFILES: DO THESE DIFFER?}

In the phase III trial that compared ESL with CBZ-CR as monotherapy for patients with newly diagnosed focal epilepsy, the safety profile of ESL was generally similar to that of CBZCR [29]. The incidence of treatment-emergent AEs judged to be at least possibly treatment- related was lower with ESL than with CBZ-CR $(42.1 \%$ vs $51.5 \%)$, as was the incidence of treatment-emergent AEs leading to discontinuation $(14.0 \%$ vs $18.4 \%)$ [29]. Fewer patients treated with ESL versus CBZ-CR experienced treatment-related increases in gamma-glutamyl transferase $(3.5 \% \quad$ vs $13.1 \%)$ and alanine aminotransferase $(0.5 \%$ vs $2.2 \%)$ [29]. In addition, fewer patients treated with ESL versus CBZ-CR discontinued because of allergic dermatitis $(0.5 \%$ vs $1.7 \%)$ [29]. The incidence of hyponatremia judged to be at least possibly treatment-related was higher with ESL than with CBZ-CR $(2.5 \%$ vs $1.0 \%)$, but only one patient (in the CBZ-CR group) discontinued because of this AE [29]. In the post hoc analysis of 1049 patients enrolled in three ESL phase III adjunctive therapy trials, in which outcomes were compared between patients who did $(n=611)$ and did not $(n=438)$ receive CBZ as a concomitant AED, the incidence of treatmentemergent AEs was higher in patients treated with CBZ (50\% with placebo; $71 \%$ with ESL) than in those who were not treated with CBZ (40\% with placebo; 55\% with ESL) [30].

Several real-world clinical practice studies have investigated the impact on tolerability of transitioning patients who were experiencing OXC- or CBZ-related AEs to ESL [31-37]. In a single-center, retrospective chart review of 23 patients with focal epilepsy who were transitioned overnight from OXC to ESL because of tolerability problems, an evaluation of the effects of the transition was made after 1 and 3 months of ESL treatment [34]. The most intolerable OXC-related AEs were somnolence, dizziness, and diplopia [34], consistent with the aforementioned pattern of OXC metabolism [19]. OXC-related AEs had resolved in 14/23 (60.9\%) and $15 / 23(65.2 \%)$ patients 1 and 3 months after transition, respectively [34]. Two-thirds (66.5\%) of OXC-related AEs occurred in the morning and $93.4 \%$ of these resolved after transitioning from OXC to ESL [34]. The only ESL-related AE reported by at least $10 \%$ of patients was headache, and, importantly, all patients continued ESL treatment throughout the study period [34].

A retrospective, single-center study was conducted in which 21 patients with drug- 
resistant focal epilepsy on a stable dose of immediate-release OXC for at least 4 weeks were transitioned overnight to ESL because they experienced persistent seizures with OXC but were unable to tolerate increased OXC dosing because of AEs [35]. Tolerability (assessed using the Adverse Events Profile [AEP]), quality of life (assessed using the Quality of Life in Epilepsy Inventory-10 [QOLIE-10]), and alertness (assessed as reaction time using a subtest of the Test Battery for Attention Performance version 2.3) were compared immediately before and 5 days after transitioning from OXC to ESL [35]. Following transition to ESL, there were significant improvements in mean scores for AEP $(p<0.001)$, QOLIE-10 $(p=0.001)$, and alertness $(p<0.05)$ [35]. Overall, AEP total scores, QOLIE10 total scores, and alertness scores improved for $100.0 \%, 81.0 \%$, and $76.2 \%$ of patients, respectively [35]. Serum sodium levels (measured on days 0 and 5) remained stable, indicating that the likelihood of developing hyponatremia did not increase after switching treatment overnight [35].

In a single-center, observational, descriptive, cross-sectional study of 61 consecutive patients with drug-resistant epilepsy who were treated with ESL over a mean follow-up duration of 4.7 months, eight of 13 patients who were transitioned overnight from CBZ to ESL experienced AEs, as did four of 12 patients who transitioned overnight from OXC to ESL, but all AEs were transient [32]. Two patients who transitioned from OXC to ESL reported that the AEs they experienced with OXC (dizziness and drowsiness) improved after transitioning to ESL [32]. Overall, decreased sodium levels were observed in 4/61 (6.6\%) patients, none of whom was taking OXC or CBZ [32]. None of these sodium values were below $125 \mathrm{mmol} / \mathrm{L}$ [32]. In addition, hyponatremia resolved (from 128 to $138 \mathrm{mmol} / \mathrm{L}$ ) in one patient and remained the same $(133 \mathrm{mmol} / \mathrm{L})$ in another patient after transitioning from OXC to ESL [32]. In a multicenter, retrospective case series of 29 elderly patients ( $>65$ years) with focal seizures who were treated with ESL, the tolerability profile improved in four of five patients who transitioned from CBZ or OXC to ESL because of AEs [36]. Overall, 3/29 (10.3\%) patients developed hyponatremia, but only one developed symptoms (confusion) that resolved after withdrawal of ESL [36].

In the EARLY-ESLI study, 14 patients converted from CBZ monotherapy to ESL monotherapy because of tolerability problems with CBZ, and only two of these patients reported AEs at 1 year [33]. In addition, of the five patients converted from OXC monotherapy to ESL monotherapy because of tolerability problems with OXC, three had no AEs with ESL at 1 year [33]. A total of 137 patients withdrew to ESL monotherapy from treatment with other AEDs during EARLY-ESLI, of whom three (2.2\%) developed hyponatremia; hyponatremia was symptomatic in only one of these patients $(113 \mathrm{mEq} / \mathrm{L})$ [33]. In the ESLIBASE study, the study population included 26 patients who were transitioned to ESL from OXC because of OXC-related AEs and 17 patients who transitioned from CBZ because of CBZ-related AEs [31]. Overall, 15/26 (57.7\%) patients previously treated with OXC no longer had AEs after transitioning to ESL, and 8/17 (47.1\%) previously treated with CBZ no longer had AEs after transitioning to ESL [31]. Thus, after transition from either OXC or CBZ to ESL, approximately $50 \%$ of patients no longer experienced AEs. In the overall ESLIBASE population, hyponatremia (ranging from 116 to $128 \mathrm{mEq} / \mathrm{L}$ ) was reported in $9 / 327(2.8 \%)$ patients and led to treatment discontinuation in four patients [31]. Hyponatremia was asymptomatic in eight patients; the remaining patient reported confusion that resolved rapidly after ESL discontinuation and did not require hospital admission [31].

Nonadherence to AEDs is a common and challenging problem in epilepsy, with nonadherence rates of up to $60 \%$ reported in some populations [37]. In the EARLY-ESLI study, adherence improved in all of the 10 patients who converted from CBZ to ESL monotherapy because of adherence problems with CBZ [33]. Similarly, of five patients who converted from OXC monotherapy to ESL monotherapy because of tolerability problems with OXC, three had no AEs with ESL at 1 year, and of three patients who converted because of adherence problems with OXC, two had no adherence problems with ESL at 1 year [33]. 


\section{Differences Within the Dibenzazepine Family in Their Impact on Lipid Markers}

Treatment with enzyme-inducing AEDs, including CBZ, has been associated with increased serum lipid levels [4]. Although (S)licarbazepine is a weak inducer of cytochrome P450 3A4 and uridine 5'-diphospho-glucuronosyltransferase [14], it is a less potent enzyme inducer than CBZ, which induces a broader range of oxidative and conjugating enzymes [1]. Lipid profiles are particularly relevant in light of epidemiological evidence demonstrating that patients with epilepsy have significantly elevated rates of cardiovascular and cerebrovascular disease [4]. In a study in which 12 patients with epilepsy transitioned from CBZ to OXC, cytochrome $\mathrm{P} 450$ enzyme system function (assessed by evaluating antipyrine pharmacokinetics) was shown to normalize after 2 months, resulting in a significant reduction in serum total cholesterol levels and a detectable but not statistically significant reduction in low-density lipoprotein cholesterol levels $(p=0.057)$, with serum concentrations of high-density lipoprotein cholesterol and triglycerides remaining unchanged [38]. Seizure control was generally similar following the transition from CBZ to $\mathrm{OXC}$, with only one patient experiencing an increase in seizure frequency [38]. Two further studies have specifically investigated the impact of ESL on patients' lipid profiles, including the effect of transitioning from CBZ or OXC to ESL $[39,40]$. A retrospective cohort study of 36 adult patients assessed lipid levels before and after initiating ESL as adjunctive therapy over an average follow-up of 11 months [39]. After at least 6 months of ESL treatment, there were significant reductions in the levels of total cholesterol (from 191.3 to $179.7 \mathrm{mg} / \mathrm{dL}$; $p<0.0001)$ and low-density lipoprotein cholesterol (from 114.6 to $103.1 \mathrm{mg} / \mathrm{dL}$; $p<0.0001$ ), and a significant increase in the level of high-density lipoprotein cholesterol (from 57.5 to $63.9 \mathrm{mg} / \mathrm{dL} ; p<0.0001$ ) [39]. A second observational, retrospective, single-center, cohort study investigated the impact of ESL treatment on the lipid profiles of 108 patients over a median duration of 23.1 months (range 3-41 months) [40]. Of these, 51.9\% had switched to ESL from prior treatment with CBZ or OXC [40]. In the overall population, mean total cholesterol levels decreased significantly from previous pathological $(>200 \mathrm{mg} / \mathrm{dL})$ to normal $(<200 \mathrm{mg} / \mathrm{dL})$ values during ESL treatment $(p=0.015)$ [40]. In the subgroup of patients who transitioned from CBZ or OXC to $\mathrm{ESL}$, there was a significant decrease in median triglyceride levels $(p<0.001)$ and mean lowdensity lipoprotein cholesterol $(p=0.014)$, and a marked decrease in mean total cholesterol $(p=0.053)$ [40]. The proportion of patients with hypercholesterolemia also decreased significantly after transitioning to ESL (from $57.1 \%$ to $37.5 \% ; p=0.018)$, as did the proportion of patients with hypertriglyceridemia (from $10.8 \%$ to $1.8 \% ; p=0.011$ ) (Fig. 3) [40]. Both studies therefore suggest that switching to ESL is of value when considering lipid profiles.

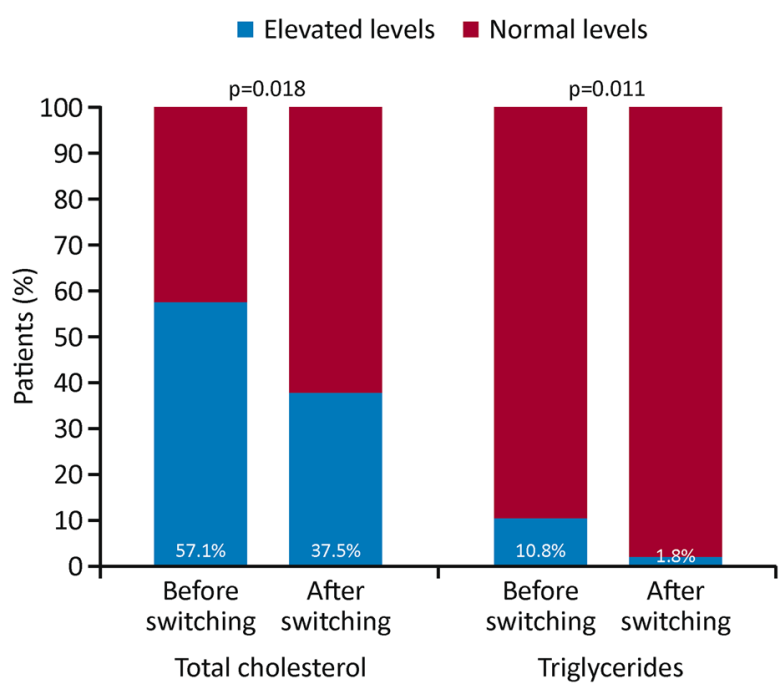

Fig. 3 Percentage of patients with normal or elevated levels of total cholesterol and triglycerides before and after switching treatment from $\mathrm{CBZ}$ or $\mathrm{OXC}$ to ESL. CBZ carbamazepine, ESL eslicarbazepine acetate, OXC oxcarbazepine. Adapted from Epilepsy Research, 115, M. Ley, A. Principe, J. Jiménez-Conde, R. Rocamora, Assessing longterm effects of eslicarbazepine acetate on lipid metabolism profile, sodium values and liver function tests, pp. 147-152, Copyright (2015), with permission from Elsevier 


\section{Tolerability Differences: Summary}

In a phase III monotherapy trial, ESL was shown to have a generally similar tolerability profile to CBZ-CR, but with lower incidences of treatment-related AEs and AEs leading to discontinuation [29]. In clinical trials of ESL adjunctive therapy, treatment regimens that included CBZ were shown to be less well tolerated than those that did not, despite attaining clinical efficacy [30]. There is also considerable evidence to demonstrate that patients transitioning from OXC or CBZ to ESL because of AEs experience improvements in tolerability [31-36]. Interestingly, improvements in quality of life and/or alertness were also reported [35]. Patients transitioning from OXC or CBZ to ESL have additionally demonstrated significant improvements in lipid profiles [40]. Transitioning from CBZ or OXC to ESL does not appear to be associated with an increased likelihood of developing hyponatremia; however, it is good practice to monitor for the potential development of hyponatremia with all dibenzazepine agents, particularly in the elderly. Blood level monitoring is not routinely required for dibenzazepine AEDs [12-14]. However, decreased platelet or white blood cell counts may occur with CBZ treatment and it is therefore recommended that a complete blood count should be obtained before starting CBZ treatment and periodically thereafter [12].

\section{CLINICAL IMPLICATIONS}

ESL has been shown to be effective in patients who have previously not achieved adequate seizure control with $\mathrm{CBZ}$ or OXC, when used instead of these agents. ESL has also been shown to improve effectiveness when added to CBZ treatment. Such evidence is consistent with findings from experimental models that have revealed differences in the mechanism of action between dibenzazepine agents [3, 27, 28], supporting the notion of "rational polytherapy", whereby agents with different mechanisms of action may synergize when used in combination to provide enhanced effectiveness [41]. Rational polytherapy must, however, also take account of the increased risk of adverse effects and pharmacokinetic interactions as a patient's drug burden is increased, and thus balance the potential benefit of increased effectiveness with polytherapy against the potential risk of increased tolerability problems [42]. The coadministration of more than one AED is likely to limit dosing of the newly added drug. In patients who fail first-line monotherapy, and have started their second monotherapy, there is evidence to support switching from CBZ or OXC to ESL. Evidence from clinical practice studies also demonstrates that there are differences in tolerability within the dibenzazepine family, since patients who have experienced CBZ- or OXC-related AEs may experience improvements in tolerability when transitioned to ESL. Furthermore, it may be appropriate to transition patients from CBZ to ESL if they are experiencing, or at risk of developing, metabolic problems resulting from $\mathrm{CBZ}$ induction of enzymes involved in endogenous metabolic pathways (e.g., hypercholesterolemia, osteoporosis, sexual dysfunction) [4, 43, 44]. Published guidance has outlined the methodological considerations associated with transitioning patients from CBZ or OXC to ESL $[43,44]$. Taken together, and despite the lack of direct comparative data, current evidence demonstrates that ESL differs from other dibenzazepine AEDs, highlighting the importance of adapting and tailoring treatment to each patient's specific needs.

\section{ACKNOWLEDGEMENTS}

Funding. Eisai Ltd funded editorial support for the preparation of this manuscript and the journal's article processing charges. All authors had full access to all of the data in this study and take complete responsibility for the integrity of the data and accuracy of the data analysis.

Authorship. All named authors meet the International Committee of Medical Journal Editors (ICMJE) criteria for authorship for this article, take responsibility for the integrity of 
the work as a whole, and have given their approval for this version to be published.

Editorial Assistance. Editorial support was provided by John Scopes of $\mathrm{mXm}$ Medical Communications and funded by Eisai Ltd.

Disclosures. Charlotte Lawthom has participated in clinical trials for Eisai; received speaker fees and participated in advisory boards for Eisai, UCB Pharma, Pfizer, GSK and Bial; and received consultancy fees from Eisai and UCB Pharma. Jukka Peltola has participated in clinical trials for Eisai, UCB and Bial; received research grants from Eisai, Medtronic, UCB and Cyberonics; received speaker honoraria from Cyberonics, Eisai, Medtronic, Orion Pharma and UCB; received support for travel to congresses from Cyberonics, Eisai, Medtronic and $\mathrm{UCB}$; and participated in advisory boards for Cyberonics, Eisai, Medtronic, UCB and Pfizer. Rob McMurray is a current employee of Eisai Europe Ltd. Emma Dodd is a current employee of Eisai Europe Ltd. Vicente Villanueva has participated in advisory boards and pharmaceutical industry-sponsored symposia for Eisai, UCB Pharma, Merck Sharp and Dohme, Bial, Pfizer, GSK, Esteve, Novartis, Medtronic, and Cyberonics.

Compliance with Ethics Guidelines. This article is based on previously conducted studies. All procedures performed in studies conducted by the authors involving human participants were in accordance with the ethical standards of the institutional and/or national research committee and with the 1964 Helsinki declaration and its later amendments or comparable ethical standards; informed consent was obtained from all individual participants included in these studies. The article also contains studies with human participants or animals that were not performed by any of the authors.

Data Availability. Data sharing is not applicable to this article as no datasets were generated or analyzed during the current study.

Open Access. This article is distributed under the terms of the Creative Commons
Attribution-NonCommercial 4.0 International License (http://creativecommons.org/licenses/ by-nc/4.0/), which permits any noncommercial use, distribution, and reproduction in any medium, provided you give appropriate credit to the original author(s) and the source, provide a link to the Creative Commons license, and indicate if changes were made.

\section{REFERENCES}

1. Brodie MJ. Sodium channel blockers in the treatment of epilepsy. CNS Drugs. 2017;31:527-34.

2. Errington AC, Stöhr T, Heers C, et al. The investigational anticonvulsant lacosamide selectively enhances slow inactivation of voltage-gated sodium channels. Mol Pharmacol. 2008;73:157-69.

3. Hebeisen S, Pires N, Loureiro AI, et al. Eslicarbazepine and the enhancement of slow inactivation of voltage-gated sodium channels: a comparison with carbamazepine, oxcarbazepine and lacosamide. Neuropharmacology. 2015;89:122-35.

4. Brodie MJ, Mintzer S, Pack AM, Gidal BE, Vecht CJ, Schmidt D. Enzyme induction with antiepileptic drugs: cause for concern? Epilepsia. 2013;54:11-27.

5. Fertig EJ, Mattson RH. Carbamazepine. In: Engel Jr J, Pedley TA, editors. Epilepsy: a comprehensive textbook, vol. 2. 2nd ed. Philadelphia: Lippincott Williams \& Wilkins; 2008. p. 1543-55.

6. Galiana GL, Gauthier AC, Mattson RH. Eslicarbazepine acetate: a new improvement on a classic drug family for the treatment of partial-onset seizures. Drugs R D. 2017;17:329-39.

7. Marson AG, Al-Kharusi AM, Alwaidh M, SANAD Study group, et al. The SANAD study of effectiveness of carbamazepine, gabapentin, lamotrigine, oxcarbazepine, or topiramate for treatment of partial epilepsy: an unblinded randomised controlled trial. Lancet. 2007;369:1000-15.

8. Shorvon S. Oxcarbazepine: a review. Seizure. 2000;9:75-9.

9. Benes J, Parada A, Figueiredo AA, et al. Anticonvulsant and sodium channel-blocking properties of novel 10,11-dihydro-5H-dibenz[b,f]azepine-5-carboxamide derivatives. J Med Chem. 1999; 42:2582-7.

10. Almeida L, Soares-da-Silva P. Eslicarbazepine acetate (BIA 2-093). Neurotherapeutics. 2007;4:88-96. 
11. Bialer M, Soares-da-Silva P. Pharmacokinetics and drug interactions of eslicarbazepine acetate. Epilepsia. 2012;53:935-46.

12. Novartis Pharmaceuticals UK Ltd. Tegretol ${ }^{\circledR}$. Summary of product characteristics; 2018. https://www. medicines.org.uk/emc/medicine/1328. Accessed 10 Sep 2018.

13. Novartis Pharmaceuticals UK Ltd. Trileptal ${ }^{\circledR}$ Summary of Product Characteristics; 2018. https://www. medicines.org.uk/emc/medicine/2673\#PHARMA COKINETIC_PROPS. Accessed 10 Sep 2018.

14. BIAL-Portela \& $C^{\mathrm{a}}$, SA. Zebinix ${ }^{\circledR}$ Summary of Product Characteristics; 2017. http://www.ema. europa.eu/docs/en_GB/document_library/EPAR_-_ Product_Information/human/000988/ WC500047225.pdf. Accessed 10 Sep 2018.

15. Hainzl D, Parada A, Soares-da-Silva P. Metabolism of two new antiepileptic drugs and their principal metabolites $S(+)$ - and $R(-)$-10,11-dihydro-10-hydroxy carbamazepine. Epilepsy Res. 2001;44: 197-206.

16. Bialer M, Johannessen SI, Levy RH, Perucca E, Tomson T, White HS. Progress report on new antiepileptic drugs: a summary of the Ninth Eilat Conference (EILAT IX). Epilepsy Res. 2009;83:1-43.

17. Patsalos PN, Stephenson TJ, Krishna S, Elyas AA, Lascelles PT, Wiles CM. Side-effects induced by carbamazepine-10,11-epoxide. Lancet. 1985;2: 1432 .

18. Mintzer S, Mattson RT. Should enzyme-inducing antiepileptic drugs be considered first-line agents? Epilepsia. 2009;50(Suppl 8):42-50.

19. Nunes T, Rocha JF, Falcão A, Almeida L, Soares-daSilva P. Steady-state plasma and cerebrospinal fluid pharmacokinetics and tolerability of eslicarbazepine acetate and oxcarbazepine in healthy volunteers. Epilepsia. 2013;54:108-16.

20. Zaccara G, Giovannelli F, Maratea D, Fadda V, Verrotti A. Neurological adverse events of new generation sodium blocker antiepileptic drugs. Meta-analysis of randomized, double-blinded studies with eslicarbazepine acetate, lacosamide and oxcarbazepine. Seizure. 2013;22:528-36.

21. Johannessen Landmark C, Svendsen T, Dinarevic J, et al. The impact of pharmacokinetic interactions with eslicarbazepine acetate versus oxcarbazepine and carbamazepine in clinical practice. Ther Drug Monit. 2016;38:499-505.

22. Gollwitzer S, Kostev K, Hagge M, Lang J, Graf W, Hamer HM. Nonadherence to antiepileptic drugs in
Germany: a retrospective, population-based study. Neurology. 2016;87:466-72.

23. Bonifácio MJ, Sheridan RD, Parada A, Cunha RA, Patmore L, Soares-da-Silva P. Interaction of the novel anticonvulsant, BIA 2-093, with voltagegated sodium channels: comparison with carbamazepine. Epilepsia. 2001;42:600-8.

24. Almeida L, Bialer M, Soares-da-Silva P. Eslicarbazepine acetate. In: Shorvon S, Perucca E, Engel J, editors. The treatment of epilepsy. 3rd ed. Oxford: Wiley-Blackwell; 2009. p. 485-98.

25. Hebeisen S, Brady K, Konrad D, Soares-da-Silva P. Inhibitory effects of eslicarbazepine acetate and its metabolites against neuronal voltage-gated sodium channels. Epilepsia. 2011;52(Suppl 6):257-8 (abstract p 851).

26. Soares-da-Silva P, Pires N, Bonifácio MJ, Loureiro AI, Palma N, Wright LC. Eslicarbazepine acetate for the treatment of focal epilepsy: an update on its proposed mechanisms of action. Pharmacol Res Perspect. 2015;3:e00124.

27. Doeser A, Soares-da-Silva P, Beck H, Uebachs M. The effects of eslicarbazepine on persistent $\mathrm{Na}^{+}$current and the role of the $\mathrm{Na}^{+}$channel $\beta$ subunits. Epilepsy Res. 2014;108:202-11.

28. Doeser A, Dickhof G, Reitze $M$, et al. Targeting pharmacoresistant epilepsy and epileptogenesis with a dual-purpose antiepileptic drug. Brain. 2015;138:371-87.

29. Trinka E, Ben-Menachem E, Kowacs PA, et al. Efficacy and safety of eslicarbazepine acetate versus controlled-release carbamazepine monotherapy in newly diagnosed epilepsy: a phase III double-blind, randomized, parallel-group, multicenter study. Epilepsia. 2018;59:479-91.

30. Halász P, Elger C, Ben-Menachem E, et al. Efficacy and safety of eslicarbazepine acetate as add-on treatment to carbamazepine in patients with partial-onset seizures. Epilepsia. 2009;50(Suppl 10):65-6 (abstract p 299).

31. Villanueva V, Serratosa JM, Guillamón E, et al. Long-term safety and efficacy of eslicarbazepine acetate in patients with focal seizures: results of the 1-year ESLIBASE retrospective study. Epilepsy Res. 2014;108:1243-52.

32. Massot A, Vivanco R, Principe A, Roquer J, Rocamora R. Post-authorisation study of eslicarbazepine as treatment for drug-resistant epilepsy: preliminary results. Neurologia. 2014;29:94-101.

33. Villanueva V, Bermejo P, Montoya J, et al. EARLYESLI study: long-term experience with 
eslicarbazepine acetate after first monotherapy failure. Acta Neurol Scand. 2017;136:254-64.

34. Mäkinen J, Rainesalo S, Peltola J. Transition from oxcarbazepine to eslicarbazepine acetate: a single center study. Brain Behav. 2017;7:e00634.

35. Schmid E, Kuchukhidze G, Kirschner M, et al. Overnight switching from oxcarbazepine to eslicarbazepine acetate: an observational study. Acta Neurol Scand. 2017;135:449-53.

36. Gómez-Ibáñez A, Serratosa JM, Guillamón E, et al. Efficacy and safety of eslicarbazepine-acetate in elderly patients with focal epilepsy: case series. Seizure. 2017;48:53-6.

37. Jones RM, Butler JA, Thomas VA, Peveler RC, Prevett M. Adherence to treatment in patients with epilepsy: associations with seizure control and illness beliefs. Seizure. 2006;15:504-8.

38. Isojärvi JI, Pakarinen AJ, Rautio A, Pelkonen O, Myllylä VV. Liver enzyme induction and serum lipid levels after replacement of carbamazepine with oxcarbazepine. Epilepsia. 1994;35:1217-20.

39. Pulitano P, Franco V, Mecarelli O, Brienza M, Davassi C, Russo E. Effects of eslicarbazepine acetate on lipid profile and sodium levels in patients with epilepsy. Seizure. 2017;53:1-3.

40. Ley M, Principe A, Jiménez-Conde J, Rocamora R. Assessing long-term effects of eslicarbazepine acetate on lipid metabolism profile, sodium values and liver function tests. Epilepsy Res. 2015;115:147-52.

41. Brodie MJ, Sills GJ. Combining antiepileptic drugs-rational polytherapy? Seizure. 2011;20: 369-75.

42. Baulac M. Rational conversion from antiepileptic polytherapy to monotherapy. Epileptic Disord. 2003;5:125-32.

43. Peltola J, Holtkamp M, Rocamora R, Ryvlin P, Sieradzan K, Villanueva V. Practical guidance and considerations for transitioning patients from oxcarbazepine or carbamazepine to eslicarbazepine acetate-expert opinion. Epilepsy Behav. 2015;50:46-9.

44. Villanueva V, Ojeda J, Rocamora RA, et al. EPICON consensus: recommendations for proper management of switching to eslicarbazepine acetate in epilepsy. Neurologia. 2018;35:290-300. 